\title{
DISTORTION THEOREMS, LIPSCHITZ CONTINUITY AND THEIR APPLICATIONS FOR BLOCH TYPE MAPPINGS ON BOUNDED SYMMETRIC DOMAINS IN $\mathrm{C}^{n}$
}

\author{
Hidetaka Hamada \\ Kyushu Sangyo University, Faculty of Science and Engineering \\ 3-1 Matsukadai, 2-Chome, Higashi-ku, Fukuoka 813-8503, Japan; h.hamada@ip.kyusan-u.ac.jp
}

\begin{abstract}
Let $\mathbf{B}_{X}$ be a bounded symmetric domain realized as the unit ball of an $n$ dimensional $\mathrm{JB}^{*}$-triple $X=\left(\mathbf{C}^{n},\|\cdot\|_{X}\right)$. In this paper, we give a new definition of Bloch type mappings on $\mathbf{B}_{X}$ and give distortion theorems for Bloch type mappings on $\mathbf{B}_{X}$. When $\mathbf{B}_{X}$ is the Euclidean unit ball in $\mathbf{C}^{n}$, this new definition coincides with that given by Chen and Kalaj or by the author. As a corollary of the distortion theorem, we obtain the lower estimate for the radius of the largest schlicht ball in the image of $f$ centered at $f(0)$ for $\alpha$-Bloch mappings $f$ on $\mathbf{B}_{X}$. Next, as another corollary of the distortion theorem, we show the Lipschitz continuity of $(\operatorname{det} B(z, z))^{1 / 2 n}|\operatorname{det} D f(z)|^{1 / n}$ for Bloch type mappings $f$ on $\mathbf{B}_{X}$ with respect to the Kobayashi metric, where $B(z, z)$ is the Bergman operator on $X$, and use it to give a sufficient condition for the composition operator $C_{\varphi}$ to be bounded from below on the Bloch type space on $\mathbf{B}_{X}$, where $\varphi$ is a holomorphic self mapping of $\mathbf{B}_{X}$. In the case $\mathbf{B}_{X}=\mathbf{B}^{n}$, we also give a necessary condition for $C_{\varphi}$ to be bounded from below which is a converse to the above result. Finally, as another application of the Lipschitz continuity, we obtain a result related to the interpolating sequences for the Bloch type space on $\mathbf{B}_{X}$.
\end{abstract}

\section{Introduction}

Let $f$ be a Bloch function on the unit disc $\mathbf{U}$ in $\mathbf{C}$. Ghatage, Yan and Zheng [6] showed that $\left(1-|z|^{2}\right)\left|f^{\prime}(z)\right|$ is Lipschitz with respect to the hyperbolic distance on $\mathbf{U}$ and used it to give a sufficient condition for the composition operator $C_{\varphi}$ to be bounded from below on the Bloch space on $\mathbf{U}$, where $\varphi$ is a holomorphic self mapping of U. Chen and Kalaj [2] generalized the above results to the Euclidean unit ball $\mathbf{B}^{n}$ in $\mathbf{C}^{n}$. They showed the Lipschitz continuity of $\left(1-\|z\|^{2}\right)^{\frac{n+1}{2 n}}|\operatorname{det} D f(z)|^{1 / n}$ for Bloch type mappings $f$ on $\mathbf{B}^{n}$ with respect to the Bergman distance on $\mathbf{B}^{n}$ and used it to give a sufficient condition for the composition operator $C_{\varphi}$ to be bounded from below on the Bloch type space on $\mathbf{B}^{n}$, where $\varphi$ is a holomorphic self mapping of $\mathbf{B}^{n}$. In the proof of Lipschitz continuity, the distortion theorem for Bloch type mappings on $\mathbf{B}^{n}$ due to Chen, Ponnusamy and Wang [3] played an important role.

Bounded symmetric domains in $\mathbf{C}^{n}$ can be realized as the unit ball $\mathbf{B}_{X}$ of a JB*-triple $X=\left(\mathbf{C}^{n},\|\cdot\|_{X}\right)$. In this paper, we give a new definition of Bloch type mappings on $\mathbf{B}_{X}$ and give distortion theorems for Bloch type mappings on $\mathbf{B}_{X}$ (Theorem 3.1). When $\mathbf{B}_{X}$ is the Euclidean unit ball in $\mathbf{C}^{n}$, this new definition coincides with that given by Chen and Kalaj [2] and the author [10]. Note that the author

https://doi.org/10.5186/aasfm.2019.4451

2010 Mathematics Subject Classification: Primary 32A18, 32M15, 47B38, 30H30.

Key words: Bloch type mapping, bounded from below, bounded symmetric domain, distortion theorem, Lipschitz continuity.

H. Hamada was partially supported by JSPS KAKENHI Grant Number JP16K05217. 
[10] (cf.[5], [13]) gave distortion theorems for other Bloch type mappings on bounded symmetric domains in $\mathbf{C}^{n}$. As in [10], [13], as a corollary of the distortion theorem, we obtain the lower estimate for the radius of the largest schlicht ball in the image of $f$ centered at $f(0)$ for $\alpha$-Bloch mappings $f$ on $\mathbf{B}_{X}$ (Theorem 3.2). Next, as another corollary of the distortion theorem, we show the Lipschitz continuity of $(\operatorname{det} B(z, z))^{1 / 2 n}|\operatorname{det} D f(z)|^{1 / n}$ for Bloch type mappings $f$ on $\mathbf{B}_{X}$ with respect to the Kobayashi metric (Theorem 4.1), where $B(z, z)$ is the Bergman operator on $X$, and use it to give a sufficient condition for the composition operator $C_{\varphi}$ to be bounded from below on the Bloch type space on $\mathbf{B}_{X}$ (Theorem 5.1), where $\varphi$ is a holomorphic self mapping of $\mathbf{B}_{X}$. In the case $\mathbf{B}_{X}=\mathbf{B}^{n}$, we also give a necessary condition for $C_{\varphi}$ to be bounded from below which is a converse to Theorem 5.1 (Theorem 5.2). Finally, as another application of the Lipschitz continuity, we obtain a result related to the interpolating sequences for the Bloch type space on $\mathbf{B}_{X}$ (Corollary 5.3).

\section{Preliminaries}

Let $X$ and $Y$ be complex Banach spaces. Let $L(X, Y)$ denote the set of continuous linear operators from $X$ into $Y$. Let $I_{X}$ be the identity in $L(X)=L(X, X)$. For a linear operator $A \in L(X, Y)$, let

$$
\|A\|_{X, Y}=\sup \left\{\|A z\|_{Y}:\|z\|_{X}=1\right\},
$$

where $\|\cdot\|_{X}$ and $\|\cdot\|_{Y}$ are the norms on $X$ and $Y$, respectively. In the case $Y=\mathbf{C}^{n}$ is the Euclidean space, we write $\|A\|_{X, e}$ for $A \in L\left(X, \mathbf{C}^{n}\right)$.

Definition 2.1. (See e.g. [4] and [17]) A complex Banach space $X$ is called a JB*-triple if $X$ is a complex Banach space equipped with a continuous Jordan triple product

$$
X \times X \times X \rightarrow X \quad(x, y, z) \mapsto\{x, y, z\}
$$

satisfying

$\left(\mathrm{J}_{1}\right)\{x, y, z\}$ is symmetric bilinear in the outer variables, but conjugate linear in the middle variable,

$\left(\mathrm{J}_{2}\right)\{a, b,\{x, y, z\}\}=\{\{a, b, x\}, y, z\}-\{x,\{b, a, y\}, z\}+\{x, y,\{a, b, z\}\}$,

$\left(\mathrm{J}_{3}\right) x \square x \in L(X)$ is a hermitian operator with spectrum $\geqq 0$,

$\left(\mathrm{J}_{4}\right)\|\{x, x, x\}\|=\|x\|^{3}$

for $a, b, x, y, z \in X$, where the box operator $x \square y: X \rightarrow X$ is defined by $x \square y(\cdot)=$ $\{x, y, \cdot\}$ and $\|\cdot\|$ is the norm on $X$.

An element $u \in X$ which satisfies $\{u, u, u\}=u$ is called a tripotent. Two tripotents $u$ and $v$ which satisfy $u \square v=0$ are said to be orthogonal. A tripotent $u$ is said to be maximal if the only tripotent which is orthogonal to $u$ is 0 .

For every $x, y \in X$, the Bergman operator $B(x, y) \in L(X)$ is defined by

$$
B(x, y)(z)=z-2(x \square y)(z)+\{x,\{y, z, y\}, x\}, \quad z \in X .
$$

When $\|x \square y\|<1$, the fractional power $B(x, y)^{r} \in L(X)$ exists for every $r \in \mathbf{R}$, since the spectrum of $B(x, y)$ lies in $\{\zeta \in \mathbf{C}:|\zeta-1|<1\}$ (cf. [14, p. 517]).

Let $\mathbf{B}_{X}$ be the unit ball of $X$. We denote by $H\left(\mathbf{B}_{X}\right)$ the family of holomorphic mappings from $\mathbf{B}_{X}$ into $X$. Let $D f(x)$ denote the Fréchet derivative of $f \in H\left(\mathbf{B}_{X}\right)$. Also, let $g_{a}: \mathbf{B}_{X} \rightarrow \mathbf{B}_{X}$ be the Möbius transformation induced by $a$, given by

$$
g_{a}(x)=a+B(a, a)^{1 / 2}\left(I_{X}+x \square a\right)^{-1}(x), \quad x \in \mathbf{B}_{X} .
$$


Then $g_{a}$ is an automorphism of $\mathbf{B}_{X}$ such that $g_{a}^{-1}=g_{-a}$, and $g_{a}(-a)=0$. We will denote by $\rho$ the Kobayashi metric on $\mathbf{B}_{X}$, which is the integral form of the infinitesimal Kobayashi metric. For $a, b \in \mathbf{B}_{X}$, we have $\rho(a, b)=\tanh ^{-1}\left\|g_{-a}(b)\right\|$, where $g_{-a}$ is the Möbius transformation induced by $-a$.

In the rest of this paper, let $\mathbf{B}_{X}$ be the unit ball of an $n$-dimensional JB*-triple $X=\left(\mathbf{C}^{n},\|\cdot\|_{X}\right)$. Also, let $h_{0}$ be the Bergman metric on $\mathbf{B}_{X}$ at 0 , and let (see [11])

$$
c\left(\mathbf{B}_{X}\right)=\frac{1}{2} \sup _{x, y \in \mathbf{B}_{X}}\left|h_{0}(x, y)\right| .
$$

The following lemma is obtained in the proof of [13, Theorem 3.1].

Lemma 2.2. Let $\alpha>0, c=c\left(\mathbf{B}_{X}\right)$ and let

$$
r(t)=t\left(1-t^{2}\right)^{c \alpha}\left(\frac{2 c \alpha+1}{2 c \alpha}\right)^{c \alpha} \sqrt{2 c \alpha+1}, \quad t \in[0,1] .
$$

Then $r(t)$ is increasing on $\left[0, \frac{1}{\sqrt{2 c \alpha+1}}\right]$, decreasing on $\left[\frac{1}{\sqrt{2 c \alpha+1}}, 1\right]$ and $r\left(\frac{1}{\sqrt{2 c \alpha+1}}\right)=1$.

The following result is a Schwarz-Pick lemma on the open unit ball $\mathbf{B}_{X}$ of the JB*-triple $X$ [7] (cf. [9, Theorem 3] and [12, Corollary 4.7]; see [8, Lemma 1.1] and [18], in the case $\mathbf{B}_{X}=\mathbf{B}^{n}$ ).

Lemma 2.3. Let $\mathbf{B}_{X}$ be the unit ball of a finite dimensional JB*-triple $X$ and let $\varphi: \mathbf{B}_{X} \rightarrow \mathbf{B}_{X}$ be a holomorphic mapping. Then

$$
|\operatorname{det} D \varphi(z)| \leq\left[\frac{\operatorname{det} B(\varphi(z), \varphi(z))}{\operatorname{det} B(z, z)}\right]^{1 / 2}, \quad \forall z \in \mathbf{B}_{X} .
$$

The above estimate is sharp and equality at a given point $z \in \mathbf{B}_{X}$ holds if and only if $\varphi$ is an automorphism of $\mathbf{B}_{X}$.

The following lemma was obtained in [10](see also [13, Lemma 2.2]).

Lemma 2.4. Let $\mathbf{B}_{X}$ be a bounded symmetric domain realized as the unit ball of an $n$-dimensional $J B^{*}$-triple $X=\left(\mathbf{C}^{n},\|\cdot\|_{X}\right)$. Then we have

$$
\operatorname{det} B(z, z) \geq\left(1-\|z\|^{2}\right)^{2 c\left(\mathbf{B}_{X}\right)}, \quad z \in \mathbf{B}_{X} .
$$

The equality holds for $z \neq 0$ such that $e=z /\|z\|$ is a maximal tripotent in $X$.

Definition 2.5. Let $\mathbf{B}_{X}$ be a bounded symmetric domain realized as the unit ball of an $n$-dimensional $\mathrm{JB}^{*}$-triple $X=\left(\mathbf{C}^{n},\|\cdot\|_{X}\right)$ and let $\alpha>0$. The prenorm $\|f\|_{\mathcal{P}(X, \alpha)}$ of $f \in H\left(\mathbf{B}_{X}\right)$ is given by

$$
\|f\|_{\mathcal{P}(X, \alpha)}=\sup _{z \in \mathbf{B}_{X}} D_{f}^{X, \alpha}(z),
$$

where

$$
D_{f}^{X, \alpha}(z)=(\operatorname{det} B(z, z))^{\alpha / 2 n}|\operatorname{det} D f(z)|^{1 / n} .
$$

Let $\mathcal{B}_{\mathcal{P}(X, \alpha)}$ be the class of all mappings $f \in H\left(\mathbf{B}_{X}\right)$ which satisfy $\|f\|_{\mathcal{P}(X, \alpha)}<$ $+\infty$. A mapping $f \in \mathcal{B}_{\mathcal{P}(X, \alpha)}$ will be called a Bloch type mapping on $\mathbf{B}_{X}$.

Remark 2.6. (i) In [13, Definition 2.5], another prenorm $\|f\|_{0, \alpha}$ is defined as follows:

$$
\|f\|_{0, \alpha}=\sup \left\{\left(1-\|z\|^{2}\right)^{\alpha c\left(\mathbf{B}_{X}\right) / n}|\operatorname{det} D f(z)|^{1 / n}: z \in \mathbf{B}_{X}\right\} .
$$

Then, by Lemma 2.4, we have $\|f\|_{0, \alpha} \leq\|f\|_{\mathcal{P}(X, \alpha)}$. 
(ii) When $\mathbf{B}_{X}$ is the Euclidean unit ball $\mathbf{B}^{n}$ in $\mathbf{C}^{n}$, then $c\left(\mathbf{B}^{n}\right)=(n+1) / 2$ ([11]) and $\operatorname{det} B(z, z)=\left(1-\|z\|^{2}\right)^{n+1}$ for all $z \in \mathbf{B}^{n}$ ([10], see also [13, Lemma 2.2]). Therefore, if $\mathbf{B}_{X}$ is the Euclidean unit ball $\mathbf{B}^{n}$ in $\mathbf{C}^{n}$, then we have $\|f\|_{0, \alpha}=\|f\|_{\mathcal{P}(X, \alpha)}$.

(iii) When $\mathbf{B}_{X}$ is the unit polydisc $\mathbf{U}^{n}$ in $\mathbf{C}^{n}$, we have $\operatorname{det} B(z, z)=\prod_{i=1}^{n}(1-$ $\left.\left|z_{i}\right|^{2}\right)^{2}$. Therefore, we have

$$
\|f\|_{\mathcal{P}(X, \alpha)}=\sup _{z \in \mathbf{U}^{n}} \Pi_{i=1}^{n}\left(1-\left|z_{i}\right|^{2}\right)^{\alpha / n}|\operatorname{det} D f(z)|^{1 / n} .
$$

When $\alpha=1,\|f\|_{\mathcal{P}(X, 1)}$ coincides with the prenorm $\|f\|_{0}$ defined by Wang and Liu [19].

As in [13, Lemma 2.6], we obtain the following lemma.

Lemma 2.7. Let $\mathbf{B}_{X}$ be a bounded symmetric domain realized as the unit ball of an $n$-dimensional $J B^{*}$-triple $X=\left(\mathbf{C}^{n},\|\cdot\|_{X}\right)$ and let $\alpha>0$.

(i) If $\|f\|_{\mathcal{P}(X, \alpha)}<+\infty$, then

$$
|\operatorname{det} D f(z)| \leq \frac{\|f\|_{\mathcal{P}(X, \alpha)}^{n}}{\left(1-\|z\|^{2}\right)^{\alpha c\left(\mathbf{B}_{X}\right)}}, \quad z \in \mathbf{B}_{X}
$$

(ii) If $\|f\|_{\mathcal{P}(X, \alpha)}=1$ and $\operatorname{det} D f(0)=1$, then $|\operatorname{det} D f(z)|=1+o(\|z\|)$.

Proof. (i) This is a consequence of the definition of $\|f\|_{\mathcal{P}(X, \alpha)}$ and Lemma 2.4.

(ii) This follows from (i).

For $x \in X \backslash\{0\}$, we define

$$
T(x)=\left\{l_{x} \in X^{*}: l_{x}(x)=\|x\|,\left\|l_{x}\right\|=1\right\},
$$

where $X^{*}$ is the dual space of $X$. Then $T(x) \neq \emptyset$ in view of the Hahn-Banach theorem. Let $H(\mathbf{U})$ denote the set of holomorphic functions on the unit disc $\mathbf{U}$ in C. The following lemma was proved by the author [10] (see also [13, Lemma 2.9]).

Lemma 2.8. Let $\mathbf{B}_{X}$ be a bounded symmetric domain realized as the unit ball of an $n$-dimensional JB*-triple $X=\left(\mathbf{C}^{n},\|\cdot\|_{X}\right)$. Let $u \in \partial \mathbf{B}_{X}$ be fixed and let

$$
f_{\psi, l_{u}}(z)=\left(\int_{0}^{l_{u}(z)} \psi(\zeta) d \zeta\right) u+z-l_{u}(z) u, \quad z \in \mathbf{B}_{X},
$$

where $l_{u} \in T(u)$ and $\psi \in H(\mathbf{U})$. Then $f_{\psi, l_{u}} \in H\left(\mathbf{B}_{X}\right), f_{\psi, l_{u}}(0)=0$ and $\operatorname{det} D f_{\psi, l_{u}}(z)=$ $\psi\left(l_{u}(z)\right)$ for $z \in \mathbf{B}_{X}$.

Let $d_{h}$ denote the hyperbolic distance on $\mathbf{U}$ :

$$
d_{h}(x, y)=\frac{1}{2} \log \frac{1+\left|\frac{x-y}{1-\bar{x} y}\right|}{1-\left|\frac{x-y}{1-\bar{x} y}\right|}=\operatorname{arctanh}\left|\frac{x-y}{1-\bar{x} y}\right|, \quad x, y \in \mathbf{U} .
$$

Denote by $D_{h}(a, r)$ the hyperbolic disc in $\mathbf{U}$ with center $a$ and radius $r$. Also, let $\mathbf{U}(a, r)=\{\zeta \in \mathbf{C}:|\zeta-a|<r\}$ for $a \in \mathbf{C}$ and $r>0$. Then the following lemma holds. The relation (i) was proved by Wang and Liu [20, Lemma 1], while the relation (ii) was recently proved by the author [10].

Lemma 2.9. Assume that $0<a<1$ and $0 \in D_{h}(a, R)$. If $g \in H(\mathbf{U})$ satisfies $g(0)=\lambda>0$ and $g\left(D_{h}(a, R)\right) \subset \mathbf{U}\left(0, \frac{\lambda}{a} \tanh R\right)$, then

(i) $\Re g(x) \geq G(x)$ for $x \in\left(0, \frac{a+\tanh (R)}{1+a \tanh (R)}\right]$ with equality for some $x$ if and only if $g=G$, where $G(\zeta)=\lambda(a-\zeta) / a(1-a \zeta) ;$ 
(ii) $|g(-x)| \leq G(-x)$ for $x \in\left(0, \frac{\tanh (R)-a}{1-a \tanh (R)}\right]$ with equality for some $x$ if and only if $g=G$.

\section{Distortion theorems}

We begin this section with the distortion theorems for Bloch type mappings $f \in \mathcal{B}_{\mathcal{P}(X, \alpha)}$. The following theorem is a generalization of [3, Theorem 1.2] to any finite dimensional bounded symmetric domain. In [13, Theorem 3.1], the following theorem was obtained under the condition $\|f\|_{0, \alpha}=1$ instead of $\|f\|_{\mathcal{P}(X, \alpha)}=1$. (When $\alpha=1$, see also [1, Theorem 2], [10, Theorem 3.1], [15, Theorem 5], [19, Theorem 3.1] and [20, Theorem 1]).

Theorem 3.1. Let $\mathbf{B}_{X}$ be a bounded symmetric domain realized as the unit ball of an $n$-dimensional $J B^{*}$-triple $X=\left(\mathbf{C}^{n},\|\cdot\|_{X}\right)$. Let $\alpha>0, \lambda \in(0,1]$, and let $m(\lambda)$ be the unique root of the equation

$$
t\left(1-t^{2}\right)^{\alpha c\left(\mathbf{B}_{X}\right)}\left(\frac{2 \alpha c\left(\mathbf{B}_{X}\right)+1}{2 \alpha c\left(\mathbf{B}_{X}\right)}\right)^{\alpha c\left(\mathbf{B}_{X}\right)} \sqrt{2 \alpha c\left(\mathbf{B}_{X}\right)+1}=\lambda
$$

in $\left[0, \frac{1}{\sqrt{2 \alpha c\left(\mathbf{B}_{X}\right)+1}}\right]$. Let $f \in \mathcal{B}_{\mathcal{P}(X, \alpha)}$ be such that $\operatorname{det} D f(0)=\lambda$ and $\|f\|_{\mathcal{P}(X, \alpha)}=1$. Then

(i)

(ii)

$$
\begin{aligned}
& \quad|\operatorname{det} D f(z)| \geq \Re \operatorname{det} D f(z) \geq \frac{\lambda}{m(\lambda)} \frac{m(\lambda)-\|z\|}{(1-m(\lambda)\|z\|)^{2 \alpha c\left(\mathbf{B}_{X}\right)+1}} \\
& \text { for }\|z\| \leq \frac{1+\sqrt{2 \alpha c\left(\mathbf{B}_{X}\right)+1} m(\lambda)}{\sqrt{2 \alpha c\left(\mathbf{B}_{X}\right)+1}+m(\lambda)}
\end{aligned}
$$

$$
\begin{gathered}
|\operatorname{det} D f(z)| \leq \frac{\lambda}{m(\lambda)} \frac{m(\lambda)+\|z\|}{(1+m(\lambda)\|z\|)^{2 \alpha c\left(\mathbf{B}_{X}\right)+1}} \\
\text { for }\|z\| \leq \frac{1-\sqrt{2 \alpha c\left(\mathbf{B}_{X}\right)+1} m(\lambda)}{\sqrt{2 \alpha c\left(\mathbf{B}_{X}\right)+1}-m(\lambda)}
\end{gathered}
$$

Proof. It suffices to use arguments similar to those in the proof of [13, Theorem 3.1] (cf. [3, Theorem 1.2], [10, Theorem 3.1]). For completeness, we give a proof for (i). Let $c=c\left(\mathbf{B}_{X}\right)$. Note that there exists a unique $m(\lambda) \in\left(0, \frac{1}{\sqrt{2 \alpha c+1}}\right]$ such that

$$
m(\lambda)\left(1-m(\lambda)^{2}\right)^{\alpha c}\left(\frac{2 \alpha c+1}{2 \alpha c}\right)^{\alpha c} \sqrt{2 \alpha c+1}=\lambda
$$

by Lemma 2.2. Let $z \in \mathbf{B}_{X} \backslash\{0\}$ be fixed and let $u=z /\|z\|$. Let

First, we consider the case $\lambda \in(0,1)$. In this case, we have $m(\lambda) \in\left(0, \frac{1}{\sqrt{2 \alpha c+1}}\right)$.

$$
g(\zeta)=(1-m(\lambda) \zeta)^{2 \alpha c} \operatorname{det} D f(\zeta u), \quad \zeta \in \mathbf{U} .
$$

Then $g \in H(\mathbf{U})$ and $g(0)=\lambda \in(0,1)$. Let $\zeta \in \mathbf{U}$ be such that $d_{h}(m(\lambda), \zeta)=$ $\operatorname{arctanh} \frac{1}{\sqrt{2 \alpha c+1}}$. This is equivalent to

$$
\left|\frac{m(\lambda)-\zeta}{1-m(\lambda) \zeta}\right|=\frac{1}{\sqrt{2 \alpha c+1}}
$$


By the relation $\|f\|_{\mathcal{P}(X, \alpha)}=1$, Lemma 2.7 (i), (3.2), (3.3) and (3.4), we have

$$
\begin{aligned}
|g(\zeta)| & \leq \frac{|1-m(\lambda) \zeta|^{2 \alpha c}\|f\|_{\mathcal{P}(X, \alpha)}}{\left(1-|\zeta|^{2}\right)^{\alpha c}}=\left(\frac{1-m(\lambda)^{2}}{1-\left|\frac{m(\lambda)-\zeta}{1-m(\lambda) \zeta}\right|^{2}}\right)^{\alpha c} \\
& =\left(1-m(\lambda)^{2}\right)^{\alpha c}\left(\frac{2 \alpha c+1}{2 \alpha c}\right)^{\alpha c}=\frac{\lambda}{m(\lambda)} \frac{1}{\sqrt{2 \alpha c+1}} .
\end{aligned}
$$

This implies that

$$
g\left(D_{h}\left(m(\lambda), \operatorname{arctanh} \frac{1}{\sqrt{2 \alpha c+1}}\right)\right) \subset \mathbf{U}\left(0, \frac{\lambda}{m(\lambda)} \frac{1}{\sqrt{2 \alpha c+1}}\right) .
$$

In view of Lemma 2.9 (i), we obtain that

$$
\Re g(\|z\|) \geq \frac{\lambda}{m(\lambda)} \frac{m(\lambda)-\|z\|}{1-m(\lambda)\|z\|}
$$

for $\|z\| \in\left(0, \frac{1+\sqrt{2 \alpha c+1} m(\lambda)}{\sqrt{2 \alpha c+1}+m(\lambda)}\right]$. By (3.3), we have

$$
\Re \operatorname{det} D f(z) \geq \frac{\lambda}{m(\lambda)} \frac{m(\lambda)-\|z\|}{(1-m(\lambda)\|z\|)^{2 \alpha c+1}},
$$

for $\|z\| \in\left(0, \frac{1+\sqrt{2 \alpha c+1} m(\lambda)}{\sqrt{2 \alpha c+1}+m(\lambda)}\right]$, i.e., we obtain the inequality $(3.1)$ for $\lambda \in(0,1)$, as desired.

Next, we consider the case $\lambda=1$. Let

$$
h(\zeta)=(1-m(1) T(\zeta))^{2 \alpha c} \operatorname{det} D f(T(\zeta) u),
$$

where

$$
T(\zeta)=\frac{m(1)(1-\zeta)}{1-m(1)^{2} \zeta}
$$

Since $T(1)=0$ and

$$
T(\mathbf{U})=\left\{w:|1-m(1) w|^{2}<1-|w|^{2}\right\} \subset \mathbf{U},
$$

the function $h$ is holomorphic on $\mathbf{U} \cup\{1\}$. Moreover, by (3.5), (3.6), Lemma 2.7 (i), and the relation $\|f\|_{\mathcal{P}(X, \alpha)}=1$, we have

$$
\begin{aligned}
|h(\zeta)| & =|1-m(1) T(\zeta)|^{2 \alpha c}|\operatorname{det} D f(T(\zeta) u)| \\
& \leq\left(1-|T(\zeta)|^{2}\right)^{\alpha c}|\operatorname{det} D f(T(\zeta) u)| \leq\|f\|_{\mathcal{P}(X, \alpha)}=1
\end{aligned}
$$

for all $\zeta \in \mathbf{U}$. Since

$$
h^{\prime}(1)=2 \alpha c(-m(1)) T^{\prime}(1)=\frac{2 \alpha c m(1)^{2}}{1-m(1)^{2}}=1
$$

by Lemma 2.7 (ii) and the relation $m(1)=1 / \sqrt{2 \alpha c+1}$, we have $h(\mathbf{U}) \subset \mathbf{U}$ by the maximum principle. Since $h(1)=1$, in view of a classical version of Julia's Lemma [16, p. 327], $h$ maps $\Delta(1, r), r>0$ of $\mathbf{U}$ into itself, where $\Delta(1, r)$ is a horodisc in $\mathbf{U}$, that is,

$$
\Delta(1, r)=\left\{z \in \mathbf{U}: \frac{|1-z|^{2}}{1-|z|^{2}}<r\right\}=\mathbf{U}\left(\frac{1}{1+r}, \frac{r}{1+r}\right) .
$$

Therefore, we have

$$
\Re h(x) \geq x, \quad \forall x \in[-1,1] .
$$


By (3.5), we have

$$
\Re \operatorname{det} D f(z) \geq \frac{1}{m(1)} \frac{m(1)-\|z\|}{(1-m(1)\|z\|)^{2 \alpha c+1}}
$$

for all $\|z\| \in[0,(1+\sqrt{2 \alpha c+1} m(1)) /(\sqrt{2 \alpha c+1}+m(1))]$, i.e., we obtain the inequality (3.1) for $\lambda=1$, as desired.

(ii) The case $\lambda=1$ is trivial. The case $\lambda \in(0,1)$ can be proved by using arguments similar those in (i). For the details, see the proof of [13, Theorem 3.1]. This completes the proof.

For $f \in H\left(\mathbf{B}_{X}\right)$, a Euclidean ball with center $f(p)$ such that $f$ maps an open subset of $\mathbf{B}_{X}$ containing $p$ biholomorphically onto this ball is called a schlicht ball of $f$ centered at $f(p)$. For a point $p \in \mathbf{B}_{X}$, let $r(p, f)$ denote the radius of the largest schlicht ball of $f$ centered at $f(p)$.

A mapping $f \in H\left(\mathbf{B}_{X}\right)$ is called an $\alpha$-Bloch mapping if

$$
\|f\|_{\alpha}<+\infty
$$

where $\|f\|_{\alpha}$ denotes the $\alpha$-Bloch semi-norm of $f$ defined by

$$
\|f\|_{\alpha}=\sup _{z \in \mathbf{B}_{X}}\left\|D f(z) B(z, z)^{\alpha / 2}\right\|_{X, e} .
$$

As a corollary of Theorem 3.1, we obtain the following lower estimate for the radius of the largest schlicht ball in the image of $f$ centered at $f(0)$ for $\alpha$-Bloch mappings $f \in H\left(\mathbf{B}_{X}\right)$. The following theorem is a generalization of [3, Theorem 1.3] to the unit ball of a finite dimensional $\mathrm{JB}^{*}$-triple. In [13, Theorem 4.5], the following theorem was obtained under the condition $\|f\|_{0, \alpha}=1$ instead of $\|f\|_{\mathcal{P}(X, \alpha)}=1$. When $\alpha=1$, it reduces to the result for Bloch mappings on bounded symmetric domains in $\mathbf{C}^{n}$ [10, Theorem 4.4] (see also [15, Theorem 6], [19, Theorem 3.3], [20, Theorem 2]). We omit the proof, since it suffices to use the arguments similar to those in the proof of [13, Theorem 4.5].

Theorem 3.2. Let $\mathbf{B}_{X}$ be a bounded symmetric domain realized as the unit ball of an $n$-dimensional JB*-triple $X=\left(\mathbf{C}^{n},\|\cdot\|_{X}\right)$ such that $\mathbf{B}_{X} \supset \mathbf{B}^{n}$, where $\mathbf{B}^{n}$ is the Euclidean unit ball of $\mathbf{C}^{n}$. Let $\alpha>0$ and $K>0$. If $f$ is an $\alpha$-Bloch mapping on $\mathbf{B}_{X}$ such that $\|f\|_{\alpha} \leq K,\|f\|_{\mathcal{P}(X, \alpha)}=1$ and $\operatorname{det} D f(0)=\lambda \in(0,1]$, then

$$
r(0, f) \geq K^{1-n} \frac{\lambda}{m(\lambda)} \int_{0}^{m(\lambda)} \frac{\left(1-t^{2}\right)^{\alpha(n-1)}(m(\lambda)-t)}{(1-m(\lambda) t)^{2 \alpha c\left(\mathbf{B}_{X}\right)+1}} d t,
$$

where $m(\lambda)$ is the unique root of the equation

$$
t\left(1-t^{2}\right)^{\alpha c\left(\mathbf{B}_{X}\right)}\left(\frac{2 \alpha c\left(\mathbf{B}_{X}\right)+1}{2 \alpha c\left(\mathbf{B}_{X}\right)}\right)^{\alpha c\left(\mathbf{B}_{X}\right)} \sqrt{2 \alpha c\left(\mathbf{B}_{X}\right)+1}=\lambda
$$

in the interval $\left[0, \frac{1}{\sqrt{2 \alpha c\left(\mathbf{B}_{X}\right)+1}}\right]$.

\section{Lipschitz continuity}

Chen and Kalaj [2, Theorem 1] obtained the Lipschitz continuity of

$$
\left(1-\|z\|^{2}\right)^{\frac{n+1}{2 n}}|\operatorname{det} D f(z)|^{1 / n}
$$


for Bloch type mappings $f$ on the Euclidean unit ball in $\mathbf{C}^{n}$ (see [6, Theorem 1] in the case $n=1$ ). The following theorem is a generalization to Bloch type mappings on bounded symmetric domains in $\mathbf{C}^{n}$.

Theorem 4.1. Let $\mathbf{B}_{X}$ be a bounded symmetric domain realized as the unit ball of an $n$-dimensional $J B^{*}$-triple $X=\left(\mathbf{C}^{n},\|\cdot\|_{X}\right)$. Let $f \in \mathcal{B}_{\mathcal{P}(X, 1)}$. Then we have

$$
\left|D_{f}^{X, 1}\left(z_{1}\right)-D_{f}^{X, 1}\left(z_{2}\right)\right| \leq M(X)\|f\|_{\mathcal{P}(X, 1)}\left[\tanh \rho\left(z_{1}, z_{2}\right)\right]^{\frac{1}{n}},
$$

for $z_{1}, z_{2} \in \mathbf{B}_{X}$, where

$$
M(X)=\left(2 c\left(\mathbf{B}_{X}\right)+1\right)^{\frac{1}{2 n}}\left(\frac{2 c\left(\mathbf{B}_{X}\right)+1}{2 c\left(\mathbf{B}_{X}\right)}\right)^{\frac{c\left(\mathbf{B}_{X}\right)}{n}} .
$$

Proof. Let $z_{1}, z_{2} \in \mathbf{B}_{X}$ be fixed. We may assume that $\|f\|_{\mathcal{P}(X, 1)}=1$ and $D_{f}^{X, 1}\left(z_{2}\right) \leq D_{f}^{X, 1}\left(z_{1}\right)$. Let $w=g_{z_{1}}^{-1}\left(z_{2}\right)$. Then, by the invariance of $\rho$ by automorphisms of $\mathbf{B}_{X}$, we have

$$
\tanh \rho\left(z_{1}, z_{2}\right)=\tanh \rho\left(g_{z_{1}}^{-1}\left(z_{1}\right), g_{z_{1}}^{-1}\left(z_{2}\right)\right)=\tanh \rho(0, w)=\|w\| .
$$

Therefore, it suffices to show that

$$
D_{f}^{X, 1}\left(z_{1}\right)-D_{f}^{X, 1}\left(z_{2}\right) \leq M(X)\|w\|^{\frac{1}{n}} .
$$

Let $F=f \circ g_{z_{1}}$. Since

$$
\left|\operatorname{det} D g_{z_{1}}(z)\right|=\left|\frac{\operatorname{det} B\left(g_{z_{1}}(z), g_{z_{1}}(z)\right)}{\operatorname{det} B(z, z)}\right|^{1 / 2},
$$

by Lemma 2.3, we have

$$
D_{F}^{X, 1}(0)=|\operatorname{det} D F(0)|^{\frac{1}{n}}=\left|\operatorname{det} D f\left(z_{1}\right)\right|^{\frac{1}{n}}\left|\operatorname{det} B\left(z_{1}, z_{1}\right)\right|^{\frac{1}{2 n}}=D_{f}^{X, 1}\left(z_{1}\right)
$$

and

$$
\begin{aligned}
D_{F}^{X, 1}(w) & =|\operatorname{det} D F(w)|^{\frac{1}{n}}|\operatorname{det} B(w, w)|^{\frac{1}{2 n}} \\
& =\left|\operatorname{det} D f\left(z_{2}\right)\right|^{\frac{1}{n}}\left|\operatorname{det} B\left(z_{2}, z_{2}\right)\right|^{\frac{1}{2 n}}=D_{f}^{X, 1}\left(z_{2}\right) .
\end{aligned}
$$

By using similar calculations, we also have $\|F\|_{\mathcal{P}(X, 1)}=1$.

If $|\operatorname{det} D F(0)|=0$, then by the inequality $D_{f}^{X, 1}\left(z_{2}\right) \leq D_{f}^{X, 1}\left(z_{1}\right)$, we have $D_{f}^{X, 1}\left(z_{2}\right)$ $=0$, Therefore, the inequality (4.1) holds. So, we may assume that $\operatorname{det} D F(0)=\lambda e^{i \theta}$ for some $\lambda \in(0,1]$ and $\theta \in[0,2 \pi]$. Let $c=c\left(\mathbf{B}_{X}\right)$ and let $m(\lambda)$ be the constant in Theorem 3.1 for $\alpha=1$. Then by (3.2), we have

$$
m(\lambda)\left(1-m(\lambda)^{2}\right)^{c} M(X)^{n}=\lambda .
$$

Case 1. Assume that $\|w\| \leq m(\lambda)$. Then, we have

$$
\left(1-\|w\|^{2}\right)^{c / n} \geq(1-\|w\| m(\lambda))^{(2 c+1) / n}
$$

and

$$
m(\lambda)^{1 / n}-\|w\|^{1 / n} \leq(m(\lambda)-\|w\|)^{1 / n} .
$$

These inequalities imply that

$$
m(\lambda)^{1 / n}-\frac{\left(1-\|w\|^{2}\right)^{c / n}(m(\lambda)-\|w\|)^{1 / n}}{(1-m(\lambda)\|w\|)^{(2 c+1) / n}} \leq\|w\|^{1 / n} .
$$

Since $m(\lambda) \leq 1$, we have

$$
\|w\| \leq m(\lambda) \leq \frac{1+\sqrt{2 c+1} m(\lambda)}{\sqrt{2 c+1}+m(\lambda)} .
$$


Also, $|\operatorname{det} D F(0)|=\lambda \leq 1$. Then, applying Theorem 3.1 to $e^{-i \theta / n} F$, we have

$$
\Re\left(e^{-i \theta} \operatorname{det} D F(w)\right) \geq \frac{\lambda}{m(\lambda)} \frac{m(\lambda)-\|w\|}{(1-m(\lambda)\|w\|)^{2 c+1}} .
$$

Therefore, by (4.2), (4.3), (4.4) and Lemma 2.4, we have

$$
\begin{aligned}
D_{f}^{X, 1}\left(z_{1}\right)-D_{f}^{X, 1}\left(z_{2}\right) & =|\operatorname{det} D F(0)|^{1 / n}-|\operatorname{det} D F(w)|^{\frac{1}{n}}|\operatorname{det} B(w, w)|^{\frac{1}{2 n}} \\
& \leq\left(\frac{\lambda}{m(\lambda)}\right)^{\frac{1}{n}}\left(m(\lambda)^{1 / n}-\frac{\left(1-\|w\|^{2}\right)^{c / n}(m(\lambda)-\|w\|)^{1 / n}}{(1-m(\lambda)\|w\|)^{(2 c+1) / n}}\right) \\
& \leq\left(\frac{\lambda}{m(\lambda)}\right)^{\frac{1}{n}}\|w\|^{\frac{1}{n}}=M(X)\left(1-m(\lambda)^{2}\right)^{\frac{c}{n}}\|w\|^{\frac{1}{n}} \\
& \leq M(X)\|w\|^{\frac{1}{n}}
\end{aligned}
$$

Case 2. Assume that $\|w\|>m(\lambda)$. Then, by (4.2), we have

$$
\begin{aligned}
D_{f}^{X, 1}\left(z_{1}\right)-D_{f}^{X, 1}\left(z_{2}\right) & \leq|\operatorname{det} D F(0)|^{1 / n}=\lambda^{1 / n} \\
& =m(\lambda)^{\frac{1}{n}}\left(1-m(\lambda)^{2}\right)^{\frac{c}{n}} M(X) \leq M(X)\|w\|^{\frac{1}{n}} .
\end{aligned}
$$

This completes the proof.

\section{Applications of the Lipschitz continuity}

As an application of Theorem 4.1, we first show a result related to a lower bound for the composition operator between the Bloch type spaces on bounded symmetric domains in $\mathbf{C}^{n}$. The following theorem is a generalization of [2, Theorem 3] to bounded symmetric domains in $\mathbf{C}^{n}$ (see [6, Theorem 2] in the case $n=1$ ).

For a holomorphic mapping $\varphi: \mathbf{B}_{X} \rightarrow \mathbf{B}_{X}$, let

$$
\tau_{\varphi}(z)=\frac{\operatorname{det} B(z, z)^{\frac{1}{2 n}}}{\operatorname{det} B(\varphi(z), \varphi(z))^{\frac{1}{2 n}}}|\operatorname{det} D \varphi(z)|^{\frac{1}{n}},
$$

where $B(z, z)$ is the Bergman operator and $n$ is the dimension of $X$. By Lemma 2.3, we always have $0 \leq \tau_{\varphi}(z) \leq 1$.

Theorem 5.1. Let $\mathbf{B}_{X}$ be a bounded symmetric domain realized as the unit ball of an $n$-dimensional JB*-triple $X=\left(\mathbf{C}^{n},\|\cdot\|_{X}\right)$. Let $\varphi$ be a holomorphic mapping of $\mathbf{B}_{X}$ into $\mathbf{B}_{X}$. Assume that there are positive constants $r$, $\varepsilon$ with $0<r<$ $M(X)^{-1}$ such that, for each $w \in \mathbf{B}_{X}$, there exists a point $z_{w} \in \mathbf{B}_{X}$ such that $\tanh \rho\left(\varphi\left(z_{w}\right), w\right)<r^{n}$ and $\tau_{\varphi}\left(z_{w}\right)>\varepsilon$. Then, we have

$$
\left\|C_{\varphi}(f)\right\|_{\mathcal{P}(X, 1)} \geq \frac{(1-r M(X)) \varepsilon}{2}\|f\|_{\mathcal{P}(X, 1)}, \quad f \in \mathcal{B}_{\mathcal{P}(X, 1)} .
$$

Proof. We may assume that $\|f\|_{\mathcal{P}(X, 1)}=1$. Then, there exists a point $w \in \mathbf{B}_{X}$ such that

$$
D_{f}^{X, 1}(w)>1-\sigma
$$

where

$$
\sigma=\frac{1-r M(X)}{2} \in(0,1 / 2) .
$$

By the assumption, there exists a point $z_{w} \in \mathbf{B}_{X}$ such that

$$
\left[\tanh \rho\left(\varphi\left(z_{w}\right), w\right)\right]^{\frac{1}{n}}<r
$$


and $\tau_{\varphi}\left(z_{w}\right)>\varepsilon$. Therefore, by applying Theorem 4.1, we have

$$
\begin{aligned}
D_{f}^{X, 1}\left(\varphi\left(z_{w}\right)\right) & \geq D_{f}^{X, 1}(w)-M(X)\left[\tanh \rho\left(\varphi\left(z_{w}\right), w\right)\right]^{\frac{1}{n}} \\
& \geq 1-\sigma-r M(X)=\frac{1-r M(X)}{2}>0 .
\end{aligned}
$$

Thus, we have

$$
\begin{aligned}
\left\|C_{\varphi}(f)\right\|_{\mathcal{P}(X, 1)} & \geq \mid \operatorname{det} D f\left(\varphi\left(z_{w}\right)\right) \operatorname{det} D \varphi\left(z_{w}\right) \operatorname{det} B\left(z_{w}, z_{w}\right)^{\left.\frac{1}{2}\right|^{\frac{1}{n}}} \\
& =D_{f}^{X, 1}\left(\varphi\left(z_{w}\right)\right) \tau_{\varphi}\left(z_{w}\right)>\frac{(1-r M(X)) \varepsilon}{2}
\end{aligned}
$$

This completes the proof.

In the case $\mathbf{B}_{X}$ is the Euclidean unit ball $\mathbf{B}^{n}$ of $\mathbf{C}^{n}$, we obtain the following theorem which is a converse to Theorem 5.1. We prepare some notations on $\mathbf{B}^{n}$. Fix $a \in \mathbf{B}^{n}$. Let

$$
P_{a}(z)= \begin{cases}\frac{\langle z, a\rangle}{\langle a, a\rangle} a, & a \neq 0, \\ 0, & a=0 .\end{cases}
$$

and let $Q_{a}=I-P_{a}$. Put $s_{a}=\left(1-\|a\|^{2}\right)^{1 / 2}$ and define the automorphism $\varphi_{a}$ of $\mathbf{B}^{n}$ by

$$
\varphi_{a}(z)=\frac{a-P_{a}(z)-s_{a} Q_{a}(z)}{1-\langle z, a\rangle} .
$$

Also, we denote $\|\cdot\|_{\mathcal{P}(X, 1)}$ by $\|\cdot\|_{\mathcal{P}(n, 1)}, \mathcal{B}_{\mathcal{P}(X, 1)}$ by $\mathcal{B}_{\mathcal{P}(n, 1)}$ and $D_{f}^{X, 1}(z)$ by $D_{f}^{n, 1}(z)$.

Theorem 5.2. Let $\varphi$ be a holomorphic mapping of $\mathbf{B}^{n}$ into $\mathbf{B}^{n}$. Assume that there exists a constant $k \in(0,1)$ such that

$$
\left\|C_{\varphi}(f)\right\|_{\mathcal{P}(n, 1)} \geq k\|f\|_{\mathcal{P}(n, 1)}, \quad f \in \mathcal{B}_{\mathcal{P}(n, 1)} .
$$

Then there exist positive constants $r, \varepsilon$ with $R<r<1$ such that, for each $w \in \mathbf{B}^{n}$, there exists a point $z_{w} \in \mathbf{B}^{n}$ such that $\tanh \rho\left(\varphi\left(z_{w}\right), w\right)<r^{n}$ and $\tau_{\varphi}\left(z_{w}\right)>\varepsilon$, where

$$
R=\left(1-k^{\frac{2 n}{n+1}}\right)^{\frac{1}{2 n}}
$$

Proof. Let $w \in \mathbf{B}^{n}$ be fixed. Let $f_{\psi, l_{u}} \in H\left(\mathbf{B}^{n}\right)$ be as in Lemma 2.8, where

$$
\psi(\zeta)=\frac{\left(1-\|w\|^{2}\right)^{\frac{n+1}{2}}}{(1-\|w\| \zeta)^{n+1}}, \quad \zeta \in \mathbf{U}
$$

and $u=\frac{w}{\|w\|}$ for $w \in \mathbf{B}^{n} \backslash\{0\}$ and $u \in \partial \mathbf{B}^{n}$ is arbitrary for $w=0$. By Lemma 2.8, we have

$$
\begin{aligned}
D_{f_{\psi, l_{u}}, 1}^{n, 1}(z) & =\left|\operatorname{det} D f_{\psi, l_{u}}(z)\right|^{\frac{1}{n}}\left(1-\|z\|^{2}\right)^{\frac{n+1}{2 n}}=\left(\frac{\left(1-\|w\|^{2}\right)\left(1-\|z\|^{2}\right)}{|1-\langle z, w\rangle|^{2}}\right)^{\frac{n+1}{2 n}} \\
& =\left(1-\left\|\varphi_{w}(z)\right\|^{2}\right)^{\frac{n+1}{2 n}}, \quad z \in \mathbf{B}^{n} .
\end{aligned}
$$

Therefore, we have $\left\|f_{\psi, l_{u}}\right\|_{\mathcal{P}(n, 1)}=1$. By the assumption, we have

$$
k=k\left\|f_{\psi, l_{u}}\right\|_{\mathcal{P}(n, 1)} \leq\left\|C_{\varphi}\left(f_{\psi, l_{u}}\right)\right\|_{\mathcal{P}(n, 1)} .
$$

Then, for any $c \in(0,1)$, there exists $z_{w} \in \mathbf{B}^{n}$ such that

$$
D_{f_{\psi, l_{u}} \circ \varphi}^{n, 1}\left(z_{w}\right) \geq c k \text {. }
$$

Since

$$
\left.D_{f_{\psi, l_{u} \circ \varphi} \circ, 1}^{n, z_{w}}\right)=\tau_{\varphi}\left(z_{w}\right) D_{f_{\psi, l_{u}}}^{n, 1}\left(\varphi\left(z_{w}\right)\right)=\tau_{\varphi}\left(z_{w}\right)\left(1-\left\|\varphi_{w}\left(\varphi\left(z_{w}\right)\right)\right\|^{2}\right)^{\frac{n+1}{2 n}}
$$


we have

$$
c k \leq \tau_{\varphi}\left(z_{w}\right)\left(1-\left\|\varphi_{w}\left(\varphi\left(z_{w}\right)\right)\right\|^{2}\right)^{\frac{n+1}{2 n}} .
$$

Since $\tau_{\varphi}\left(z_{w}\right) \leq 1$, we have

$$
c k \leq \tau_{\varphi}\left(z_{w}\right)
$$

and

$$
c k \leq\left(1-\left\|\varphi_{w}\left(\varphi\left(z_{w}\right)\right)\right\|^{2}\right)^{\frac{n+1}{2 n}} .
$$

Let

$$
r=\left(1-(c k)^{\frac{2 n}{n+1}}\right)^{\frac{1}{2 n}}
$$

and let $\varepsilon=c k$. Then we have $r \in(R, 1)$,

$$
\tanh \rho\left(\varphi\left(z_{w}\right), w\right)=\left\|\varphi_{w}\left(\varphi\left(z_{w}\right)\right)\right\| \leq r^{n}
$$

and $\tau_{\varphi}\left(z_{w}\right) \geq \varepsilon$. This completes the proof.

As another application of Theorem 4.1, we obtain the following result related to the interpolating sequences for the Bloch type space on bounded symmetric domains in $\mathbf{C}^{n}$. A sequence $\left(z_{\nu}\right)$ in $\mathbf{B}_{X}$ is said to be separated in the Kobayashi metric if there exists a constant $\varepsilon>0$ such that $\rho\left(z_{\nu}, z_{\mu}\right) \geq \varepsilon$ whenever $\nu \neq \mu$. The following corollary generalizes [6, Corollary 1]. We omit the proof, since it suffices to use arguments similar to those in the proof of [6, Corollary 1].

Corollary 5.3. Let $\mathbf{B}_{X}$ be a bounded symmetric domain realized as the unit ball of an $n$-dimensional JB*-triple $X=\left(\mathbf{C}^{n},\|\cdot\|_{X}\right)$. If a sequence $\left\{z_{\nu}\right\} \subset \mathbf{B}_{X}$ satisfies the property that the map $S: \mathcal{B}_{\mathcal{P}(X, 1)} \rightarrow \ell^{\infty}$ defined by $S(f)=\left\{\operatorname{det} D f\left(z_{\nu}\right) \operatorname{det} B\left(z_{\nu}, z_{\nu}\right)^{\frac{1}{2}}\right\}$ is onto, then $\left\{z_{\nu}\right\}$ is separated in the Kobayashi metric.

\section{References}

[1] Bonk, M., D. Minda, and H. Yanagihara: Distortion theorem for Bloch functions. - Pacific J. Math. 179, 1997, 241-262.

[2] Chen, S., and D. KalaJ: Lipschitz continuity of Bloch type mappings with respect to Bergman metric. - Ann. Acad. Sci. Fenn. Math. 43, 2018, 239-246.

[3] Chen, S., S. Ponnusamy, and X. Wang: Landau-Bloch constants for functions in $\alpha$-Bloch spaces and Hardy spaces. - Complex Anal. Oper. Theory 6, 2012, 1025-1036.

[4] Chu, C.-H.: Jordan structures in geometry and analysis. - Cambridge Tracts in Math. 190, Cambridge Univ. Press, Cambridge, 2012.

[5] Chu, C.-H., H. Hamada, T. Honda, and G. Kohr: Distortion of locally biholomorphic Bloch mappings on bounded symmetric domains. - J. Math. Anal. Appl. 441, 2016, 830-843.

[6] Ghatage, P., J. Yan, and D. Zheng: Composition operators with closed range on the Bloch space. - Proc. Amer. Math. Soc. 129, 2001, 2039-2044.

[7] Graham, I., H. Hamada, G. Kohr, and M. Kohr: Loewner chains, Bloch mappings and Pfaltzgraff-Suffridge extension operators on bounded symmetric domains. - Submitted.

[8] Graham, I., G. Kohr, and J. A. Pfaltzgraff: Parametric representation and linear functionals associated with extension operators for biholomorphic mappings. - Rev. Roum. Math. Pures Appl. 52, 2007, 47-68.

[9] Hahn, K. T.: Subordination principle and distortion theorems on holomorphic mappings in the space $\mathbf{C}^{n}$. - Trans. Amer. Math. Soc. 162, 1971, 327-336.

[10] Hamada, H.: A distortion theorem and the Bloch constant for Bloch mappings in $\mathbf{C}^{n}$. - J. Anal. Math. (to appear), https://doi.org/10.1007/s11854-019-0005-y. 
[11] Hamada, H., T. Honda, and G. Kohr: Trace-order and a distortion theorem for linearly invariant families on the unit ball of a finite dimensional JB*-triple. - J. Math. Anal. Appl. 396, 2012, 829-843.

[12] Hamada, H., and G. Kohr: Pluriharmonic mappings in $\mathbf{C}^{n}$ and complex Banach spaces. J. Math. Anal. Appl. 426, 2015, 635-658.

[13] Hamada, H., and G. Kohr: $\alpha$-Bloch mappings on bounded symmetric domains in $\mathbf{C}^{n}$. Complex Anal. Oper. Theory 12, 2018, 509-527.

[14] Kaup, W.: A Riemann mapping theorem for bounded symmetric domains in complex Banach spaces. - Math. Z. 183, 1983, 503-529.

[15] LiU, X. Y.: Bloch functions of several complex variables. - Pacific J. Math. 152, 1992, 347-363.

[16] LiU, X. Y., and D. Minda: Distortion theorems for Bloch functions. - Trans. Amer. Math. Soc. 333, 1992, 325-338.

[17] Loos, O.: Bounded symmetric domains and Jordan pairs. - University of California, Irvine, 1977.

[18] Rudin, W.: Function theory in the unit ball of $\mathbf{C}^{n}$. - Springer-Verlag, New York, 1980.

[19] WANG, J., and T. LiU: Bloch constant of holomorphic mappings on the unit polydisk of $\mathbf{C}^{n}$. - Sci. China Ser. A 51, 2008, 652-659.

[20] Wang, J., and T. LiU: Distortion theorem for Bloch mappings on the unit ball $\mathcal{B}^{n}$. - Acta Math. Sin. (Engl. Ser.) 25, 2009, 1583-1590.

Received 6 November 2018 • Accepted 17 January 2019 